\title{
Decentralized Voltage Regulation in Islanded DC Microgrids Based on a Modified Droop Control using Superimposed AC Voltage
}

\author{
Mohammadreza Nabatirad, Graduate Student Membe, IEEE, Reza Razzaghi, Member, IEEE, and Behrooz \\ Bahrani, Senior Member, IEEE,
}

\begin{abstract}
The conventional droop control is a widely-used technique in load sharing among Distributed Generator (DG) units in islanded DC Microgrids (MGs). This method provides Plug-and-Play (PnP) capability for DG units; however, poor load sharing accuracy and unregulated voltage are two shortcomings of that. This article proposes a novel control system in islanded DC MGs to provide simultaneous regulated voltage and accurate load sharing. The method utilizes a modified droop control technique in a decentralized manner. The proposed control system injects a superimposed AC voltage to the network that carries a frequency proportional to the master DG unit output current. The injected voltage adjusts an added a term to the conventional droop control named as the voltage compensation term in order to cancel voltage changes. This term adjusts terminal voltage of DG units proportional to the frequency of the superimposed AC voltage. The performance of the proposed control system is validated via a set of simulation studies using PLECS, and the experimental results confirm the viability and feasibility of the proposed control system.
\end{abstract}

Index Terms-DC Microgrid, Decentralized Control, Load Sharing, Voltage Regulation.

\section{INTRODUCTION}

$\mathbf{U}$ TILIZATION of Renewable Energy Sources (RES) has recently become an inevitable approach to tackle the climate change issue. In this respect, Distributed Generator (DG) units are considered as one of the solutions to avoid challenges regarding expansion of the current power system while getting benefits of RES [1]. Microgrids (MGs) are known as an interface between RES, DG units, and the bulk power system in the presence of Energy Storage Systems (ESSs) [2]. Due to the significant advances in power conversion technologies specifically in DC applications, DC MGs are receiving more attention compared to $\mathrm{AC}$ ones as 1) most of the RES conversion units and ESSs are basically DC-coupled components and 2) the DC MG solution is efficient and appropriate for small distribution systems such as data centres, space explorations, electric vehicles, and shipboard power systems [3]-[6].

Energy resources in DC MGs are classified as non-dispatchable units like PV systems and dispatchable units like ESS-based DG units. This classification rationalizes the importance of load sharing and voltage regulation in islanded DC MGs [7]. Power generation-demand balance must be satisfied in an islanded DC MG. In addition to the regulation of the network voltage, converters need to share load with respect to their ratings to avoid overloading failures. Accurate load sharing and regulated voltage in islanded DC MGs are recognized as two determinative factors for the DC MG performance evaluation [8]. Communicationbased approaches in control of islanded DC MGs are typically

The authors are with the department of Electrical and Computer Systems Engineering, Monash University, Clayton, Australia. Corresponding author: Mohammadreza Nabatirad, email: mohammadreza.nabatirad@monash.edu prone to single-point failure, which leads to declined reliability [9]. Additionally, in such approaches, the plug-and-play ( $\mathrm{PnP})$ capability of DG units, as an essential attribute in MGs, is not featured [10].

Among non-communication-based load sharing methods presented in the literature for islanded DC MGs [11], [12], droop control is a well-known method aiming to share loads among dispatchable DG units through controlling the terminal voltage of DG units [13]-[15]. This technique utilizes a virtual resistance as a droop coefficient to control the terminal voltage. One of the advantages of using droop control is that this control method is implemented in a fully decentralized manner. On the other hand, this load sharing technique does not include the impact of line impedance in load sharing. Thus, the voltage drop across the lines is not taken into account, which causes load sharing inaccuracy [16]. Thus, in the conventional droop control, there is a trade-off between voltage regulation and load sharing accuracy .

Modifications in the conventional droop control have been suggested to achieve simultaneous regulated voltage and accurate load sharing [13], [17]-[20]. These control techniques mostly rely on low bandwidth communication, and the non-communicationbased modified control techniques partially improve the load sharing accuracy and voltage regulation as simultaneous goala [19]. Within this context, the idea of superimposing an AC voltage on the DC voltage in a DC MG is proposed in [21]-[23] to address the mentioned shortcomings of the conventional droop control. In these studies, the generated reactive or active power by DG units (depending on the structure of the distribution lines) is used for load sharing in a decentralized manner. However, the effects caused by the network loads are not considered. In addition, to measure the injected reactive and active power by the converters, the AC current is filtered. This task is challenging and requires highly precise measurement devices possessing high resolution analog to digital converters as the amplitude of the AC current is negligible, and the converter switching ripple can affect this signal. Also, the voltage is regulated by droop gain adjustments which makes the dynamic response slow compared to a fixed DC voltage supply for the DC MG. Overall, this control system develops complexity to be implemented, and the dependency of the load sharing technique on distribution line characteristics reduces its accuracy.

An alternative technique based on the concept of conventional droop control and the superimposed AC voltage is proposed in this article. In this technique, the DC voltage of the network carries a superimposed AC component injected by a master DG unit whose frequency is dependent on the network loading. Other DG units within the MG act as slave or non-dispatchable DG units and detect the frequency of the superimposed $\mathrm{AC}$ voltage to achieve simultaneous load sharing and voltage regulation. In addition, PnP 


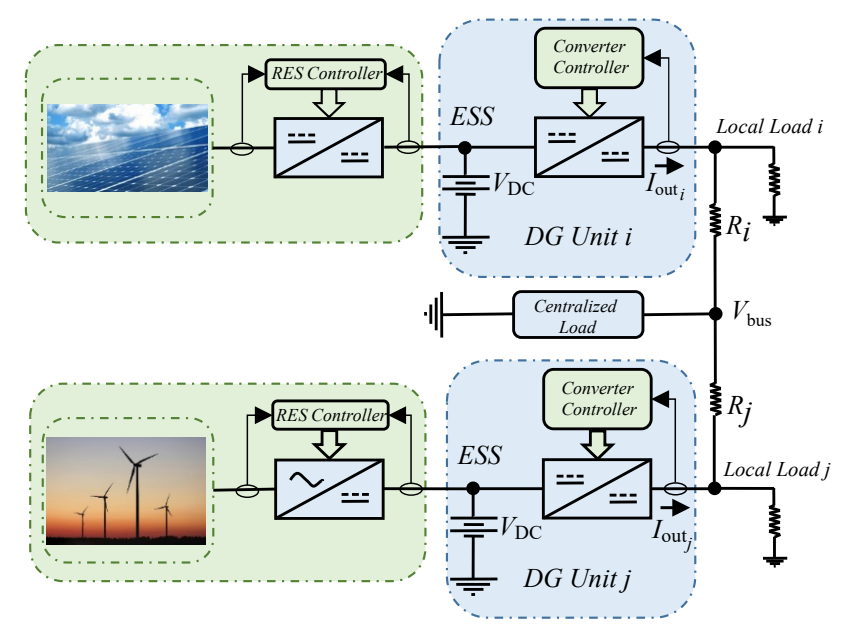

Fig. 1. An autonomous DC MG.

feature is provided for the DC MG, and non-communication based control system raises the reliability of the network performance. As a result, the proposed control system provides decentralized voltage regulation for DC MGs, and accurate load sharing as an essential term in stable operation of autonomous DC MGs is achieved. The main contribution of this paper is to introduce an easy-to-implement decentralized control strategy for DC MGs, which is not dependent on the distribution line parameters, and is simple for tuning compared to the existing techniques.

This rest of this article is organized as follows. In Section II, load sharing and its importance in DC MGs are presented. The proposed control system for load sharing and voltage regulation is introduced in Section III. Section IV evaluates the performance of the proposed technique via simulation and experimental results. Finally, the conclusions are provided in Section V.

\section{LOAD SHARING IN DC MICROGRIDS}

Load sharing and stable operation of the islanded network are closely related to each other. Usually, the output current of DG units is considered as a means of evaluating the proportion of carrying the network load. It is highly probable to utilize DG units with different ratings in a DC MG. In addition, the network topology might affect the portion of supplied power by DG units. Thus, accurate load sharing matters inevitably.

In droop-controlled DC MGs, by controlling the terminal voltages of DG units, loads are shared. The droop coefficient applies a voltage drop at each loading conditions of the network to the terminal of the DG unit using its output current as a feedback term. In this regard, the terminal voltage of each DG unit is controlled as a linear function of the output current. this function is shown as

$$
V_{\text {out }}=V_{\mathrm{n}}-K_{V I} \times I_{\text {out }},
$$

where

- $V_{\text {out }}=$ the DG unit terminal voltage,

- $V_{\mathrm{n}}=$ the DG unit nominal voltage,

- $K_{V I}=$ the V-I Droop coefficient,

- $I_{\text {out }}=$ the DG unit output current.

Fig. 1 shows an autonomous DC MG, which comprises two DC DG units supplying local loads an a centralized load. In the conventional droop control, the droop coefficients act as virtual resistances for their corresponding DG units. Therefore, by inspecting the network configuration shown in Fig. 1, the lines resistance, $R_{i}$ and $R_{j}$, participate in load sharing too. Assuming no local loads for the network, the total resistance of the $i^{\text {th }}$ and $j^{\text {th }}$ DG units are

$$
\begin{aligned}
& R_{i_{\text {total }}}=K_{V I_{i}}+R_{i}, \\
& R_{j_{\text {total }}}=K_{V I_{j}}+R_{j} .
\end{aligned}
$$

Assuming the same nominal voltage for both DG units, the output current of each DG unit is proportional to the combination of the line resistance and the droop coefficient inversely. This proportional relation for two DG units is

$$
\frac{I_{\mathrm{out}_{i}}}{I_{\mathrm{out}_{j}}}=\frac{R_{j}+K_{V I_{j}}}{R_{i}+K_{V I_{i}}},
$$

where $R_{i}$ and $R_{j}$ demonstrate line resistances, and $K_{V I_{i}}$ and $K_{V I_{j}}$ demonstrate droop coefficients of the $i^{t h}$ and $j^{t h}$ DG, respectively. Finally the output currents of DG units are $I_{i}$ and $I_{j}$. In order to reduce the negative impact of line resistances, large droop coefficients are needed, although it will cause a large and to some extend unacceptable voltage drop according to (1). It should be noted that in the literature the voltage profile following any dynamic changes has been suggested as $\pm 5 \%$ of the nominal voltage of the network [24]. Considering this fact, the droop coefficient of each DG unit equipped with the conventional voltage-droop control should be defined as

$$
K_{V I_{i}}=\frac{\Delta V_{\max }}{I_{i, \max }}, \forall i,
$$

where $\Delta V_{\max }$ is the maximum allowable voltage variation with respect to the nominal value, and $I_{i_{\text {max }}}$ is the maximum output current of the DG unit. To increase load sharing accuracy, larger droop coefficients are preferred. However, the voltage drop should be compensated to be maintained within the range. Voltage shifting techniques are the solution to dynamically shift the voltage back to its predefined nominal value in such situations [8]. Mostly, voltage shifting techniques employ distributed communications, which are based on the combination of decentralized and distributed control [8], [13], [25]. The modified droop control by the voltage shifting technique using a voltage compensation term is

$$
V_{\text {out }}=V_{\mathrm{n}}-K_{V I} \times I_{\text {out }}+\left(\Delta V_{\text {out }}\right) .
$$

In this paper, the proposed control system employs the compensation term in (6) to provide simultaneous accurate load sharing and voltage regulation in a decentralized way.

\section{Proposed Control System In DC Microgrids}

To achieve simultaneous accurate load sharing and regulated voltage in DC MGs in a decentralized manner, the proposed control system employs the modified droop control. The proposed control system utilizes a superimposed $\mathrm{AC}$ voltage injected by a master DG unit on the DC voltage. The other dispatchable DG units known as slave DG units use this signal to adjust their compensation term. The master DG unit generates a fixed DC voltage level and is equipped with a frequency droop control, while the slave DG units use a modified voltage droop control and a synchronization unit. The application of the proposed control system in a DC MG with both dispatchable and non-dispatchable DG units and integration of DC MGs into AC power systems is explained in [26] and [27] in details. The proposed control system for the master DG and slave DG units are introduced in this section. 


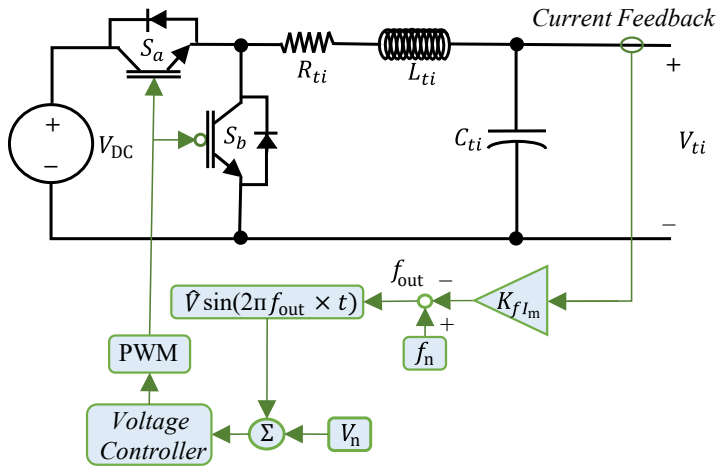

Fig. 2. Master DG unit control system structure.

\section{A. Master DG Unit Control System}

The superimposed AC voltage is generated by the master DG unit in the proposed technique, and the rest of DG units synchronize themselves with this AC voltage. The master unit provides a fixed DC voltage at its terminal regardless of the loading. Additionally, the master unit injects a superimposed AC voltage in addition to its DC value at its terminal whose frequency is proportional to its loading, i.e., its output current. Fig. 2 shows the proposed control system structure for the master DG unit. In Fig. 2, the master DG unit parameters are defined as

- $V_{\mathrm{DC}}=$ the DC source voltage,

- $K_{f I_{\mathrm{m}}}=$ the frequency-droop coefficient for the master unit,

- $f_{\mathrm{n}}=$ the nominal frequency of the superimposed voltage,

- $R_{t i}=$ the filter resistance of the converter,

- $L_{t i}=$ the filter inductance of the converter,

- $C_{t i}=$ the filter capacitance of the converter,

- $V_{\mathrm{n}}=$ the DG unit nominal voltage,

- $\hat{V}=$ the amplitude of the superimposed voltage.

To make the frequency of the superimposed AC voltage clearly detectable by a PLL, it should be at least ten times less than the switching frequency of the converter. Additionally, the range of the frequency drop needs to be limited to have proper filtering and PLL performance. Therefore, the acceptable range is $\pm 10 \%$ of $f_{\mathrm{n}}$ in order to adjust the PLL for synchronizing the slave units. $K_{f I_{\mathrm{m}}}$ is calculated as

$$
K_{f I_{\mathrm{m}}}=\frac{\Delta f_{\max }}{I_{\mathrm{m}, \text { max }}},
$$

where $\Delta f_{\max }$ is the maximum allowable frequency variation, and $I_{\mathrm{m}, \max }$ is the output current of the master DG unit while operating at full-load condition. In this study, $0.5 \%$ of the nominal voltage of the network is chosen as the amplitude of the superimposed AC voltage, which is in the range of the acceptable voltage variation. It should be noted that this amplitude is a fixed value, and the network loading does not have any impacts on it.

\section{B. Slave DG Units Control System and Synchronization}

The modified droop control is utilized in slave DG units, which requires a voltage compensation voltage. Fig. 3 shows the control system structure of the slave units. Without applying the proposed voltage compensation method, the terminal voltage would vary with respect to the output current, i.e. loading. Adding to the parameters of the DC/DC converter in Fig. 2, $V_{\text {Net }}$ is the network voltage after the synchronization switch for slave DG units as shown in Fig. 3.

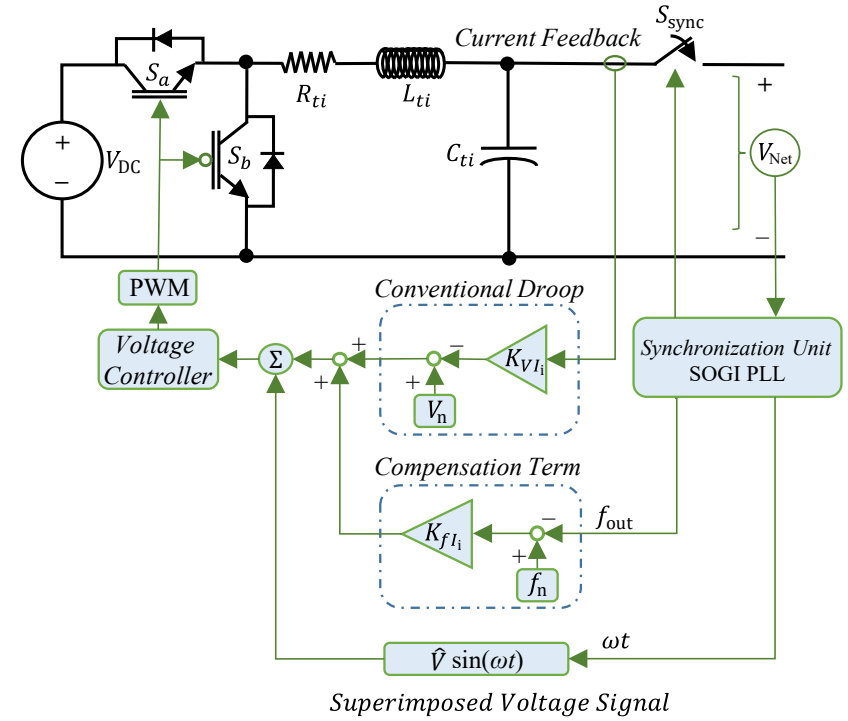

Fig. 3. Slave DG unit control system structure.

As illustrated in Fig. 3, the conventional droop control is modified by a voltage compensation term. This term is adjusted by comparing the nominal predefined frequency with the frequency of the superimposed AC voltage. More frequency drop represents more loading on the master unit. Therefore, the compensation term adjusts the terminal voltage considering the frequency deviation. Thus, the modified droop control for the slave units is defined as

$$
V_{\text {out }_{i}}=V_{\mathrm{n}}-K_{V I_{i}} \times I_{\text {out }_{i}}+\left[\left(K_{f I_{i}} \times\left(f_{\mathrm{n}}-f_{\text {out }}\right)\right] .\right.
$$

The proportional relation of the output currents of DG units controlled by the proposed control system is

$$
\frac{I_{\mathrm{out}_{\mathrm{m}}}}{I_{\mathrm{out}_{i}}}=\frac{R_{i}+K_{V I_{i}}}{R_{\mathrm{m}}+\left(K_{f I_{\mathrm{m}}} \times K_{f I_{i}}\right)},
$$

where the subscript $m$ represents the master unit. As the network voltage is fixed by the master unit, the droop and frequency coefficients do not influence the voltage. The slave DG units droop coefficients can take large values compared to the lines resistance. Therefore, accurate load sharing is achievable.

The proposed control system is expected to provide $\mathrm{PnP}$ feature. To achieve this, slave DG units must be plugged after being synchronized with the AC superimposed signal. In this regard, a synchronization unit is employed in the control system of each DG unit which is responsible for extracting the phase and frequency of the superimposed AC voltage. The synchronization unit is equipped with a single-phase PLL relying on an Orthogonal Signal Generator (OSG)-Second Order Generalized Integrator (SOGI) [28].

The general structure of the single-phase PLL equipped with the OSG-SOGI structure is shown in Fig. 4. The normalization blocks are essential to improve the accuracy of the PLL. The output signals are the network frequency $\left(f_{\text {out }}\right)$ for voltage compensation term in the modified droop control, and $\omega t$ is the angular velocity of the superimposed voltage for synchronization. $H_{d}(s)$ and $H_{q}(s)$ are defined as

$$
\begin{aligned}
& H_{d}(s)=\frac{v^{\prime}}{V_{\mathrm{AC}}}(s)=\frac{k \omega s}{s^{2}+k \omega s+\omega^{2}}, \\
& H_{q}(s)=\frac{q v^{\prime}}{V_{\mathrm{AC}}}(s)=\frac{k \omega^{2}}{s^{2}+k \omega s+\omega^{2}},
\end{aligned}
$$




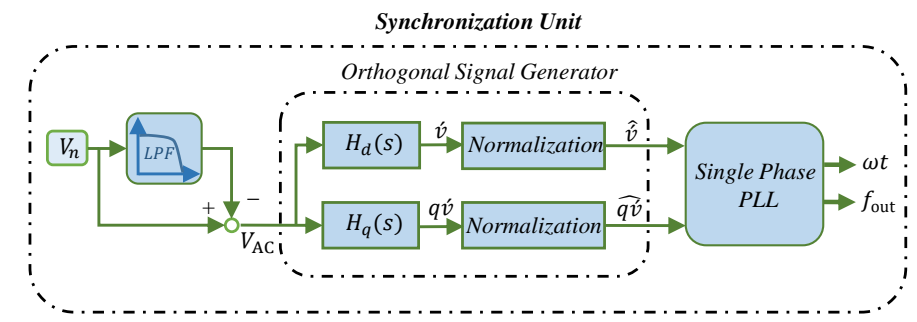

Fig. 4. Structure of the synchronization unit and PLL.

where $\omega$ represents the resonance frequency of the SOGI, and $k$ is the gain that affects the bandpass of the OSG-SOGI structure. To have accurate frequency measurement, low frequency bandpass across the resonance frequency should be employed. However, due to the frequency deviation with respect to the master DG unit output current, high bandpass is desired. For this compromise and according to the design criteria of the DC MG in power rating of DG units and loading condition, $k$ needs to be set. In this study, $k$ is set to be 0.8 , and the resonance frequency is $190 \pi \mathrm{rad} / \mathrm{sec}$. This set of parameters allows $\pm 5 \mathrm{~Hz}$ frequency deviation across $95 \mathrm{~Hz}$.

\section{Stability Analysis}

In order to analyze the stability of the network two major disturbances in an islanded DC MG are taken into study; load change and DG unit rating change. The DG unit rating change represents connecting or disconnecting a DG unit to the network. A DC MG composed of two DG units equipped with the proposed control system is considered. State Space (SS) model of the mentioned DC MG is derived by applying Kirchhoff voltage and current laws through the network. Taking into account Fig. 2 and Fig. 3 , in the DC MG, signals $V_{t i}, V_{\mathrm{DC}}, I_{t_{i}}, I_{L_{i}}$ and $I_{\mathrm{Line}_{i}}$ are the load voltage at the point of common coupling (PCC), the DC source voltage, the filter current, the local load current, and the distribution line current, respectively. The equations of DG units output current and terminal voltage are

$$
\mathrm{DG}_{i}:\left\{\begin{array}{ll}
\frac{d V_{i}}{d t}= & \frac{1}{C_{t i}} I_{t i}+\frac{1}{C_{t i}} I_{\mathrm{Line}_{i j}}-\frac{1}{C_{t i}} I_{L i} \\
\frac{d I_{t i}}{d t}= & -\frac{R_{t i}}{L_{t i}} I_{t i}-\frac{1}{L_{t i}} V_{i}+\frac{1}{L_{t i}} V_{t i}
\end{array} .\right.
$$

It is assumed that $I_{\operatorname{Line}_{i j}}(t)=-I_{\text {Line }_{j i}}(t), \forall t>0$. In low voltage DC MGs, $L_{\text {Line }_{i j}}$ is small enough to be neglected, i.e. $\frac{d I_{\text {Line }_{i j}}}{d t}=$ 0 . This fact is valid by exploiting quasi-stationary line (QSL) approximation of the distribution lines [29]. The SS equations of a DC MG according to (12) are

$$
\begin{array}{r}
\dot{x}_{g_{i}}=A_{g_{i i}} x_{g_{i}}+\sum_{j \in N_{i}} A_{g_{i j}} x_{g_{i}}+B_{g_{i}} u_{i}, \\
y_{i}=C_{g_{i}} x_{g_{i}} ; i=1, \cdots, N,
\end{array}
$$

where $x_{g_{i}}=\left[V_{i}, I_{t_{i}}\right]^{T}$ are the state variables, $u_{i}$ includes the normalized input voltage $V_{\mathrm{DC}}$ and the local load current $I_{L_{i}}$, and $y_{i}=V_{i}$ is the output voltage (terminal voltage) of $\mathrm{DG}_{i}$. The SS matrices are defined as

$$
\begin{gathered}
A_{g_{i i}}=\left[\begin{array}{cc}
-\sum_{j \in N_{i}} \frac{1}{C_{t_{i}} R_{i j}} & \frac{1}{C_{t i}} \\
-\frac{1}{L_{t i}} & -\frac{R_{t i}}{L_{t i}}
\end{array}\right], \\
A_{g_{i j}}=\left[\begin{array}{cc}
\frac{1}{C_{t_{i}} R_{i j}} & 0 \\
0 & 0
\end{array}\right],
\end{gathered}
$$

(a)

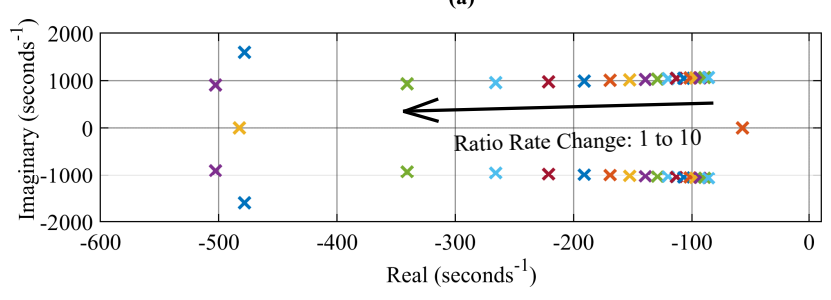

(b)

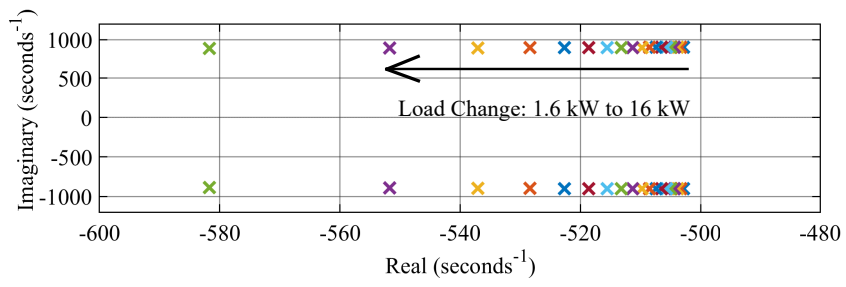

Fig. 5. Place of dominant poles of the network equipped with the proposed control system; (a) rating ratio and (b) local load change.

$$
\begin{gathered}
B_{g_{i}}=\left[\begin{array}{cc}
0 & -\frac{1}{C_{t_{i}}} \\
\frac{1}{L_{t i}} & 0
\end{array}\right], \\
C_{g_{i}}=\left[\begin{array}{ll}
1 & 0
\end{array}\right] .
\end{gathered}
$$

The subscript $i$ for the master unit is set as m, and the subscript $j$ represents the variables related to the other DGs connected to $\mathrm{DG}_{i}$ starting from two. The term $\sum_{j \in N_{i}} A_{g_{i j}} x_{g_{i}}$ shows the coupling between $\mathrm{DG}_{i}$ and its connections.

The proposed control system affects the output voltage of the slave units. In the first step, the input vector $u$, for a DC MG with two DGs, should be rearranged as

$$
u=u_{0}+k \times x
$$

where $u_{0}$ contains fixed values, and $k$ represents the connection matrix of state variables and the input vector. As a result,

$$
u=\left[\begin{array}{llll}
V_{\mathrm{DC}} & \frac{V_{\mathrm{m}}}{R_{L_{\mathrm{m}}}} & V_{\mathrm{DC}}-K_{V I_{2}} I_{2}+K_{f I_{2}} \Delta f & \frac{V_{2}}{R_{L_{2}}}
\end{array}\right]^{T} .
$$

where $\Delta f$ is the frequency deviation read by the slave unit. Considering (19), the new state matrices of the mentioned DC MG are derived as

$$
\begin{aligned}
& A_{g_{\mathrm{mm}}}=\left[\begin{array}{cc}
-\left(\frac{1}{C_{t_{\mathrm{m}}}}\right)\left(\frac{1}{R_{m 2} 2}+\frac{1}{R_{L_{\mathrm{m}}}}\right) & \frac{1}{C_{t \mathrm{~m}}} \\
-\frac{1}{L_{t \mathrm{~m}}} & -\frac{R_{t \mathrm{~m}}}{L_{t \mathrm{~m}}}
\end{array}\right], \\
& A_{g_{22}}=\left[\begin{array}{cc}
-\left(\frac{1}{C_{t_{2}}}\right)\left(\frac{1}{R_{2 m}}+\frac{1}{R_{L_{2}}}\right) & \frac{1}{C_{t 2}} \\
-\frac{1}{L_{t 2}} & \left(-\frac{1}{L_{t 2}}\right)\left(R_{t 2}+K_{V I_{2}}\right)
\end{array}\right] \text {, } \\
& A_{g_{2 \mathrm{~m}}}=\left[\begin{array}{cc}
\frac{1}{C_{t_{2}} R_{2 m}} & 0 \\
0 & \left(-\frac{1}{L_{t 2}}\right)\left(K_{f I_{\mathrm{m}}} K_{f I_{2}}\right)
\end{array}\right] .
\end{aligned}
$$

By modifying state matrices, the input vector for the mentioned DC MG is equal to

$$
u_{0}=\left[\begin{array}{llll}
V_{\mathrm{DC}} & 0 & V_{\mathrm{DC}} & 0
\end{array}\right]^{T} .
$$

The stability of the network is analyzed with respect to local load and DG units ratio $\left(K_{V I_{2}}\right)$ variations. To this end, for a two-DG DC network with typical network and DG parameters, the rating proportion of the master unit versus the slave unit is changed from 1 to 10. Fig. 5(a) illustrates system poles for this DC MG. In addition, the slave unit local load resistance is varied 


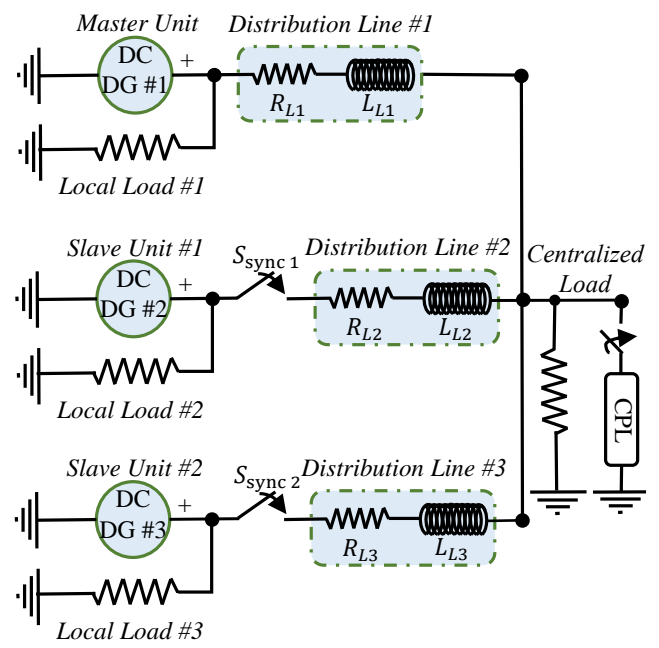

Fig. 6. Under-study DC MG.

TABLE I

DG UNITS AND NETWORK PARAMETERS IN THE SIMULATION

\begin{tabular}{c||c||c||c} 
Parameters & DG \#1 (Master) & DG \#2 (Slave) & DG \#3 (Slave) \\
\hline \hline$V_{\mathrm{DC}}$ & $410 \mathrm{~V}$ & $410 \mathrm{~V}$ & $410 \mathrm{~V}$ \\
$V_{\mathbf{n}}$ & $400 \mathrm{~V}$ & $400 \mathrm{~V}$ & $400 \mathrm{~V}$ \\
$f_{s w}$ & $10 \mathrm{kHz}$ & $10 \mathrm{kHz}$ & $10 \mathrm{kHz}$ \\
$L_{f}$ & $0.8 \mathrm{mH}$ & $0.8 \mathrm{mH}$ & $0.8 \mathrm{mH}$ \\
$R_{f}$ & $0.02 \Omega$ & $0.02 \Omega$ & $0.02 \Omega$ \\
$C_{f}$ & $500 \mu \mathrm{F}$ & $500 \mu \mathrm{F}$ & $500 \mu \mathrm{F}$ \\
$P_{\mathbf{n}}$ & $6000 \mathrm{~W}$ & $3000 \mathrm{~W}$ & $1500 \mathrm{~W}$ \\
$K_{V I}, K_{f I}$ & $\mathrm{~N} / \mathrm{A}, \mathrm{N} / \mathrm{A}$ & $\Omega, 8 \mathrm{~V} / \mathrm{Hz}$ & $8 \Omega, 4 \mathrm{~V} / \mathrm{Hz}$ \\
$K_{f I_{\mathbf{m}}}$ & $0.5 \mathrm{~Hz} / \mathrm{A}$ & $\mathrm{N} / \mathrm{A}$ & $\mathrm{N} / \mathrm{A}$ \\
$f_{\mathbf{n}}$ & $100 \mathrm{~Hz}$ & $100 \mathrm{~Hz}$ & $100 \mathrm{~Hz}$ \\
$\hat{V}_{\mathrm{AC}}$ & $2 V_{\text {peak }}$ & $2 V_{\text {peak }}$ & $2 V_{\text {peak }}$ \\
$R_{L}, L_{L}$ & $0.15 \Omega, 5 \mu H$ & $0.35 \Omega, 9 \mu H$ & $0.25 \Omega, 7 \mu \mathrm{H}$ \\
$R_{\text {Local }}$ & $200 \Omega$ & $200 \Omega$ & $200 \Omega$ \\
& & &
\end{tabular}

from $100 \Omega$ to $10 \Omega(1.6 \mathrm{~kW}$ to $16 \mathrm{~kW})$ for a fixed slave unit rating. For this case, the root loci of the DC MG is shown in Fig. 5(b). It should be noted that considering practical limitations, the loading of the network should always be coherent with the rating of the DG units and the voltage level. For this test, it is assumed that the rating of the DC MG is large enough to supply a $16 \mathrm{~kW}$ load.

According to the shown results in Fig. 5, by increasing network loading and decreasing nominal rating of the slave units, the stability margin increases. In other words, more reliance on the master unit, increases the stability margin by moving dominant poles to far left side of the imaginary axis.

\section{Performance Evaluation}

A PLECS model is developed to evaluate the performance of the proposed control system. Additionally, an experimental setup is configured to experimentally validate the findings.

\section{A. Simulation Results}

A DC MG comprising three DG units is modelled for the simulation studies. In this model, DG \#1 acts as the master DG unit, and the rest of the DG units are slave units. Fig. 6 illustrates the structure of the under-study islanded DC MG for the simulation studies. According to the features of the proposed control system, more DG units can be employed without imposing any changes in the parameters or communication between the DG units. The parameters of the DG units and the network are listed in Table I. The nominal frequency of the superimposed AC voltage is set at $100 \mathrm{~Hz}$. However, note that the performance of the proposed control system is not dependent on this value. In case any other source in the system generates $100 \mathrm{~Hz}$ frequency component, e.g., double frequency component in a single-phase AC system, other nominal frequencies, e.g., $50 \mathrm{~Hz}$ or $150 \mathrm{~Hz}$, can be used.

According to (7) and the PLL design criteria for synchronization and frequency measurement, the maximum allowable frequency variation is limited. The OSG-SOGI PLL gain, $k$, is set to be one, and the resonance frequency is $190 \pi \mathrm{rad} / \mathrm{sec}$. This set of parameters allows $\pm 5 \mathrm{~Hz}$ frequency deviation across $95 \mathrm{~Hz}$. Therefore, for this case $10 \mathrm{~Hz}$ is set as $\Delta f_{\max }$. The maximum output current of the master DG unit is $15 A$. Therefore, $K_{f I_{\mathrm{m}}}$ should be set less than $0.6 \mathrm{~Hz} / A$. Accordingly, the frequency droop for the master DG unit is set as $0.5 \mathrm{~Hz} / A$. For the slave DG units, the proportional current ratio in (9) should be satisfied. In (9), $K_{V I}$ should be large enough compared to the distribution line resistance to maintain load sharing accuracy. In this study, $K_{V I}$ of the slave DG units are set as $8 \Omega$ which cause neglecting the effect of distribution line resistances, as a result $K_{f I}$ for slave DG units considering predefined power ratings and ratios should be $8 \mathrm{~V} / \mathrm{Hz}$ for the first slave DG unit and $4 \mathrm{~V} / \mathrm{Hz}$ for the second one.

The simulation studies on the proposed control system are split into two sections. The first section is dedicated to synchronization of the slave units and monitoring the output currents to test the PnP capability of the DG units in the network. The second section studies the centralized Constant Power Loads (CPL) connection to the network as a disturbance analysis.

1) PnP Capability of DG Units - Synchronization: In this test, the PnP capability of the DG units is tested. The MG initially consists of only the master DG unit. The nominal voltage of the network is $400 \mathrm{~V}$, and the the peak of the superimposed AC voltage is $0.5 \%$ of the nominal voltage, which is negligible compared to the nominal DC value. The frequency of the superimposed voltage is $100 \mathrm{~Hz}$. At $\mathrm{t}=0.7 \mathrm{~s}$ and at $\mathrm{t}=1 \mathrm{~s}$, the first and second slave units are connected to the network, respectively. As stated previously, DG \#1, acts as the master DG unit and energizes the network before the slave units connection. Fig. 7(a) and (b) show the DC bus voltage of the DC MG and the output current of the DG units. Also, Fig. 7(c) demonstrates the value of the voltage compensation term for the slave DG units.

As expected, the master DG unit provides a fixed DC voltage for the network with a superimposed AC component. The network voltage after connecting the slave DG units proves that no changes in the voltage is imposed on the network and confirms accurate voltage regulation. In addition, it is needed to inspect output currents of DG units to evaluate load sharing accuracy. As shown in Fig. 7(b), before connecting the slave DG units to the network, the master DG unit supplies its local and the centralized load, which is a $1 \mathrm{~kW}$ resistive load. Other DG units are in charge of supplying their own local loads. After connecting the first slave DG unit to the network, the total loading of the network is shared between the master DG unit and slave DG unit \#1 according to the defined ratings. The simulation results confirm that the master DG unit generates $4.36 \mathrm{~A}$, and the first slave DG unit carries $2.19 \mathrm{~A}$, which is almost half of the master one. Then, by adding the second slave DG unit, accurate load sharing is still satisfied. The master DG unit injects $4.84 A$, the first slave DG unit injects $2.44 A$, and the second slave DG unit is in charge of injecting $1.28 A$ to the 
(a)

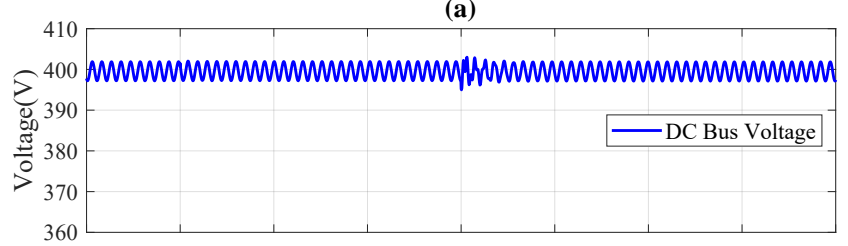

(b)

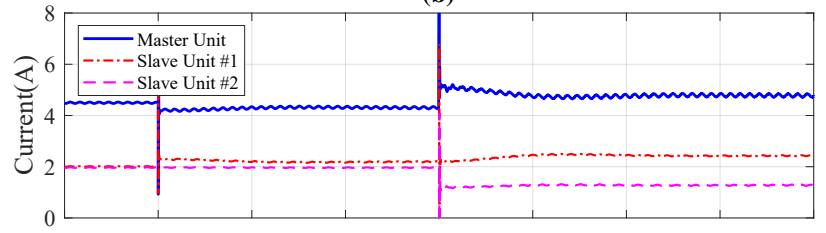

(c)

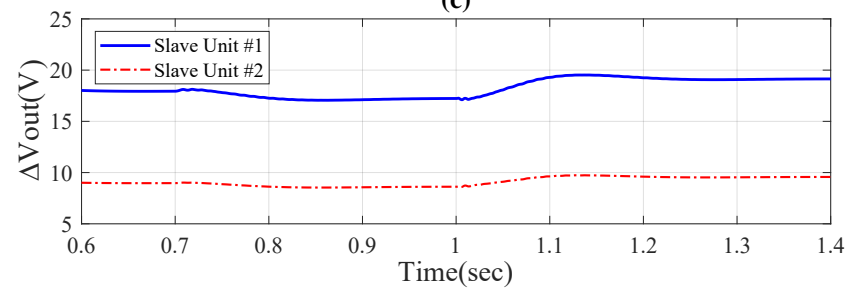

Fig. 7. Simulation results of the proposed control system while connecting the slave units: (a) DC Bus voltage, (b) output currents of DG units, and (c) compensation voltage of the slave DG units.

(a)

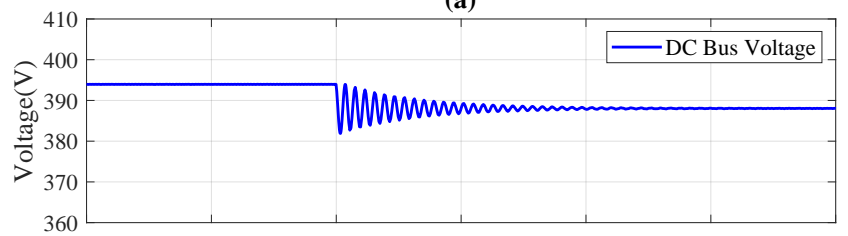

(b)

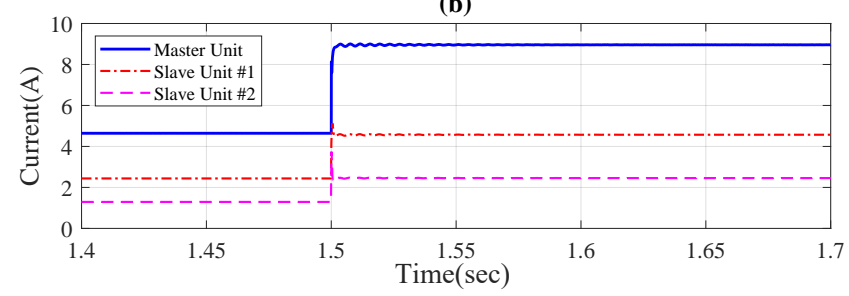

Fig. 8. Simulation results of the conventional droop control in the presence of load change: (a) DC Bus voltage of the DC MG and (b) output currents of the DG units.

network. Thus, simultaneous accurate load sharing and regulated voltage is achieved using the proposed control system. According to Fig. 7(c), the voltage compensation value $\left(\Delta V_{\text {out }}\right)$ is linearly proportional to the the frequency droop coefficient of the slave DG units.

2) Load Change - CPL Connection: In the following, first, the conventional droop control is tested on the DC MG. Then, the same test is repeated for the proposed control system. It is assumed that all DG units are equipped with the conventional droop control with respect to their ratings.

a) Conventional Droop Control: In the DC network shown in Fig. 6, the first slave DG unit has half of the rating of the master DG unit while the second slave DG unit's rating is a quarter of the master DG unit. The droop coefficient for DG \#1 is selected 1.2 $\Omega$. In addition, it is assumed that RESs are capable of keeping ESS always fully charged, and $V_{\mathrm{DC}}$ is a fixed value. The local loads are purely resistive, and the centralized load is a fixed $1 \mathrm{~kW}$ resistive load and a variable $3 \mathrm{~kW}$ CPL.

As PnP capability is featured in the conventional droop control

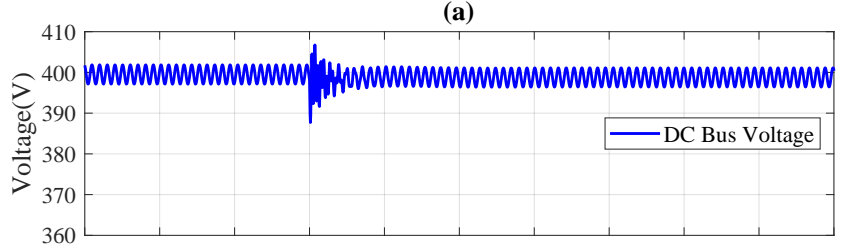

(b)

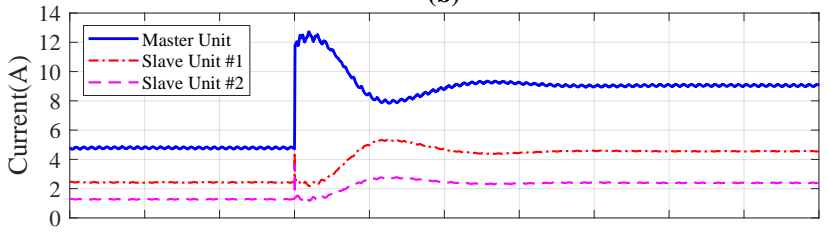

(c)

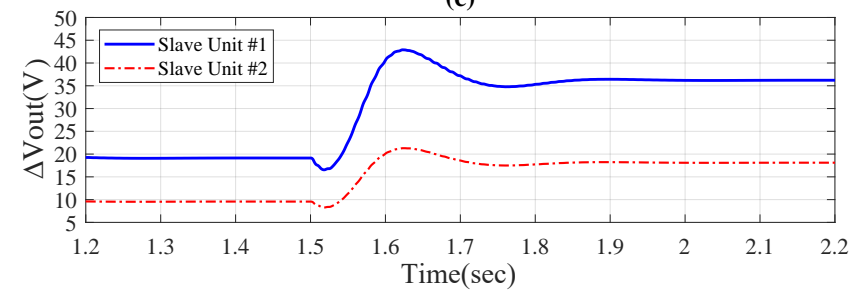

Fig. 9. The simulation results of the proposed control system in the presence of the centralized CPL connection: (a) DC Bus voltage of the DC MG, (b) the output currents of the DG units, and (c) the compensation voltage of the slave DG units.

system, a load change and effects of this disturbance on voltage and load sharing are taken under consideration. At $\mathrm{t}=1.5 \mathrm{~s}$, the centralized load (CPL) is connected. Fig. 8 shows the simulation results of the network in the presence of the load change. By applying the conventional droop control, the terminal voltages of the DG units after connecting the CPL present damped oscillations, and the network voltage in this case drops to less than $390 \mathrm{~V}$. Additionally, as shown in Fig. 8(b), load sharing is not accurate. DG \#1 injects almost $8.9 A$ to the network, while DG \#3 injects $2.4 A$ in the steady state, which is not proportional to the defined ratings.

b) The Proposed Control System: To assess the performance of the control system subsequent to a load change, a CPL is added to the network at $t=1.5 \mathrm{~s}$. Fig. 9 illustrates the simulation results of the output voltage and current of the DG units.

The network voltage is expected to be fixed. As shown in Fig. 9(a), the network voltage experiences negligible transient upon the connection of the centralized load. Fig. 9(b) depicts the DG units output currents. The damped low frequency oscillation of the output currents is due to the PLL response affected by the network elements such as distribution lines. In steadystate and subsequent to the load change, the master DG unit is injecting almost $9.12 A$ to the network, while the first slave DG unit carries half of this value that is almost equal to $4.57 \mathrm{~A}$, and the second slave unit injects $2.38 \mathrm{~A}$. Hence, load sharing accuracy and regulated voltage have been achieved concurrently. In Fig. 9(c), the voltage compensation value of the slave DG units is shown. As expected and similar to the DG connection section, the compensation voltages are proportional to the frequency droop coefficients.

3) Master DG Unit Failure: The master DG unit plays an important role in the proposed control system. As previously stated, one of the DG units in the DC MG should act as the master DG unit. However, outage of this DG unit should not stop the DC MG from operation. In this regard, a slave DG unit is controlled 
(a)

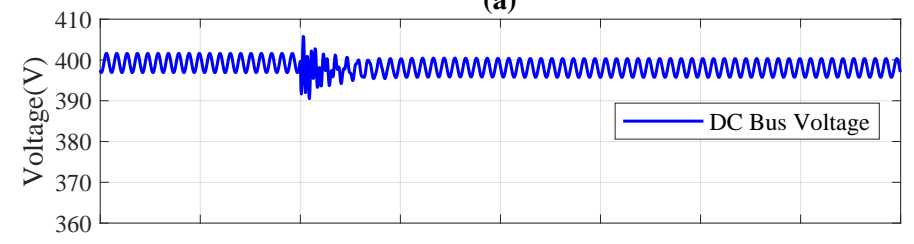

(b)

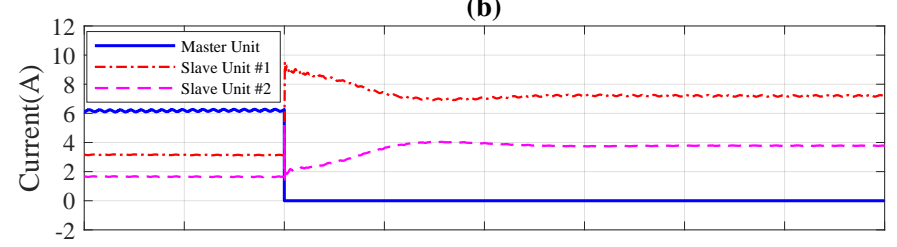

(c)

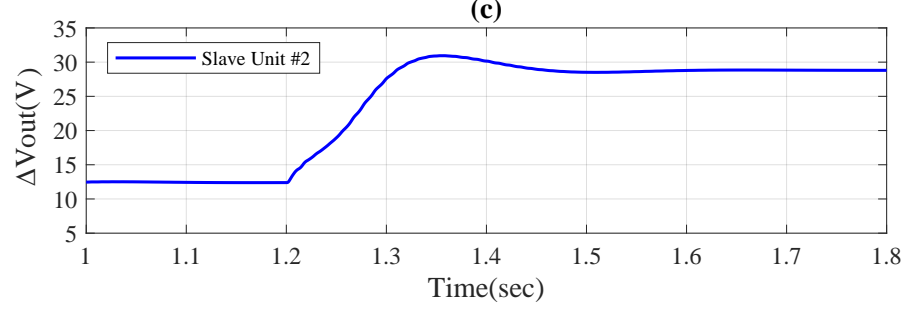

Fig. 10. The simulation results of the proposed control system in the presence of the master DG unit outage: (a) DC Bus voltage of the DC MG, (b) the output currents of the DG units, and (c) the compensation voltage of the slave DG unit \#2.

to be a redundant master DG unit. If the master DG unit fails, the nominated slave DG unit changes its role to a new master unit. This task is done by inspecting the terminal voltage of the nominated slave unit and the frequency of the superimposed AC voltage. One of the advantages of the proposed control system is the regulated DC bus voltage. In addition, the frequency deviation range is limited for the slave DG units. According to (7) and in a case the master DG unit fails, the frequency drop will be more than $8 \mathrm{~Hz}$, and the voltage compensation term in the modified droop control will get saturated. In addition, according to (8) and due to the fact that the DG units' nominal voltage $\left(V_{\mathrm{n}}\right)$ is close to the primary DC source voltage $\left(V_{\mathrm{DC}}\right)$ for the DC/DC buck converters, the network voltage does not remain regulated. If the master DG unit fails and the network is not overloaded, in addition to the frequency drop and the voltage compensation term saturation, the network voltage ultimately rises to $V_{\mathrm{DC}}$ without any superimposed $\mathrm{AC}$ voltage. This change of voltage is used as a signal for the redundant master unit to change its role. Fig. 10 depicts the simulation results upon the outage of the master DG unit.

In this part of the simulation study, the loading of the DC MG is decreased to avoid overloading the rest of DG units when the master DG unit is out. Fig. 10(a) shows the voltage of the DC bus while the master DG unit gets disconnected at $\mathrm{t}=1.2 \mathrm{~s}$. The first slave DG unit plays the role of the redundant master unit for the network. As shown in Fig. 10(b), after the outage, the network load is shared among the remaining DG units accurately. The redundant slave DG unit generates $7.3 \mathrm{~A}$, and the second slave DG unit injects 3.7 A to the network. The voltage compensation term of the second slave DG unit is shown in Fig. 10(c). This term is not saturated, and the network continues its normal operation with the proposed control system reliably.

\section{B. Experimental Results}

Based on the practical limitations, the ratings of the DC MG is reduced to implement the experimental setup. The experimental

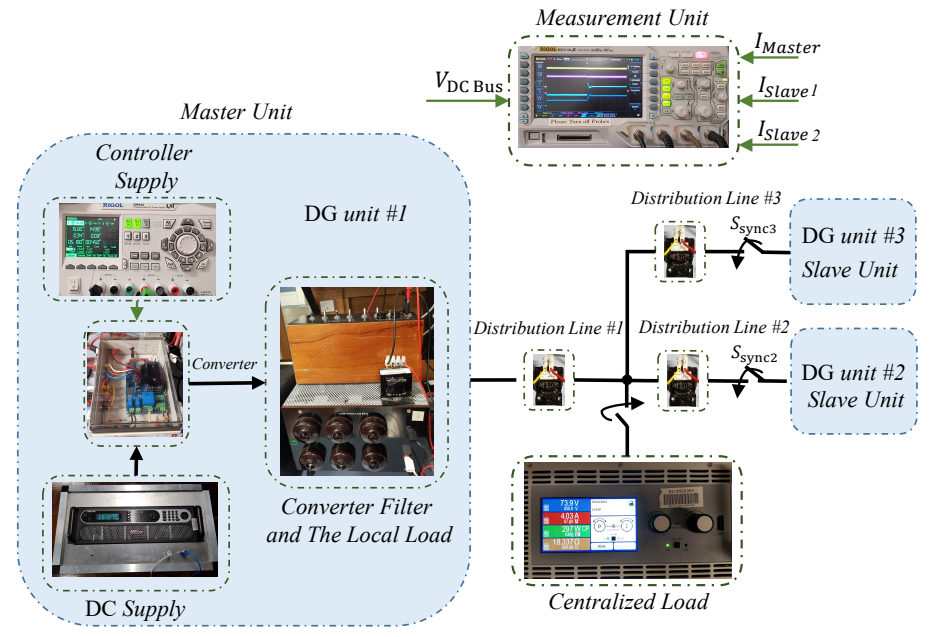

Fig. 11. Schematic diagram of the experimental setup.

setup forms an islanded DC MG comprising three DG units. The first DG unit is the master DG unit, and other DG units act as the slave DG units. Table II presents the parameter values of the experimental setup. The centralized load is a digital controllable electric load that is set as a $300 \mathrm{~W}$ CPL. A Digital Signal Processor (TMS320F28379D) is the controller for each converter. Fig. 11 shows the schematic diagram of the experimental setup. Three test cases are studied in this section: 1) PnP capability test via connecting the DG unit \#3,2) disturbance rejection via connecting a CPL, and 3) the master DG unit outage for redundancy test.

1) PnP Capability: In this experiment, the second slave DG unit is connected to the network at a specific time to test $\mathrm{PnP}$ feature of the proposed control system. Before connecting this DG unit, the master DG unit energizes the network and shares the network load with the first slave DG unit. The centralized load is a $300 \mathrm{~W}$ CPL. The second slave unit just supplies its local load. At t=0.6 s, $S_{\text {sync } 3}$ turns on, and the second slave unit gets connected to the network. Fig. 12 shows the terminal voltage and output current of the DG units.

As expected and similar to the simulation results and according to Fig. 12 (a), after connecting the second slave DG unit to the network, the DC bus voltage of the DC MG remains fixed at $75 \mathrm{~V}$, and the output currents are proportional to the ratings. As shown in Fig. 12 (b) The master DG unit injects almost $5.13 A$, the first slave DG unit generates $2.74 \mathrm{~A}$, and DG unit \#3 injects $1.40 \mathrm{~A}$ to the network. Fig. 12 (c) represents the captured frequency by the first slave DG unit. The superimposed frequency rises from $94 \mathrm{~Hz}$ to almost $95 \mathrm{~Hz}$ due to less loading on the master DG unit. This test proves PnP capability of the DG units.

2) Load Change - CPL Connection: In this test, all DG units are supplying their local loads, and the centralized load turns on. A step change in the load demand is imposed on the DC MG. The centralized load is a $300 \mathrm{~W}$ CPL, which is expected to be shared between the DG units proportionally. While the DC MG is operating in steady state, at $t=0.6 \mathrm{~s}$, the CPL turns on. Figs. 13 (a) and (b) illustrate the DC bus voltage of the network and DG units output current, respectively, upon the connection of the load. According to the aforementioned specifications, the $300 \mathrm{~W}$ CPL should be supplied by all DG units in a decentralized manner. The output current of the DG units are accurately proportional to 
TABLE II

EXPERIMENTAL SETUP PARAMETERS.

\begin{tabular}{c||c||c||c} 
Parameters & DG \#1 (Master) & DG \#2 (Slave) & DG \#3 (Slave) \\
\hline \hline$V_{\mathrm{DC}}$ & $100 \mathrm{~V}$ & $100 \mathrm{~V}$ & $100 \mathrm{~V}$ \\
$V_{\mathbf{n}}$ & $75 \mathrm{~V}$ & $75 \mathrm{~V}$ & $75 \mathrm{~V}$ \\
$f_{\text {sw }}$ & $20 \mathrm{kHz}$ & $20 \mathrm{kHz}$ & $20 \mathrm{kHz}$ \\
$L_{f}$ & $0.8 \mathrm{mH}$ & $0.8 \mathrm{mH}$ & $15 \mathrm{mH}$ \\
$C_{f}$ & $20 \mu \mathrm{F}$ & $20 \mu \mathrm{F}$ & $30 \mu \mathrm{F}$ \\
$P_{\mathbf{n}}$ & $450 \mathrm{~W}$ & $225 \mathrm{~W}$ & $150 \mathrm{~W}$ \\
$K_{V I}, K_{f I}$ & $\mathrm{~N} / \mathrm{A}, \mathrm{N} / \mathrm{A}$ & $4 \Omega, 2 \mathrm{~V} / \mathrm{Hz}$ & $4 \Omega, 1 \mathrm{~V} / \mathrm{Hz}$ \\
$K_{f I_{\mathbf{m}}}$ & $1 \mathrm{~Hz} / \mathrm{A}$ & $\mathrm{N} / \mathrm{A}$ & $\mathrm{N} / \mathrm{A}$ \\
$f_{\mathbf{n}}$ & $100 \mathrm{~Hz}$ & $100 \mathrm{~Hz}$ & $100 \mathrm{~Hz}$ \\
$\hat{V}_{\mathrm{AC}}$ & $2 V_{\text {peak }}$ & $2 V_{\text {peak }}$ & $2 V_{\text {peak }}$ \\
$R_{L}, L_{L}$ & $0.2,400 \mu H$ & $0.2 \Omega, 400 \mu H$ & $0.1 \Omega, 56 \mu H$ \\
$R_{\text {Local }}$ & $28 \Omega$ & $28 \Omega$ & $500 \Omega$
\end{tabular}

(a)

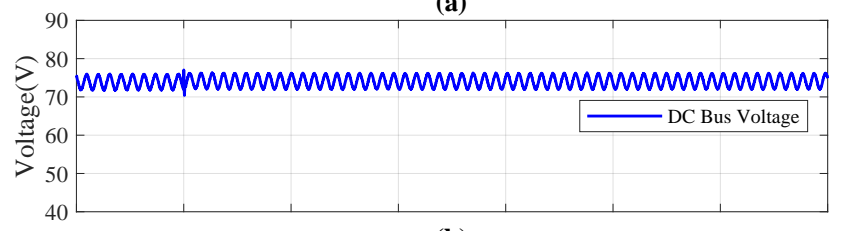

(b)

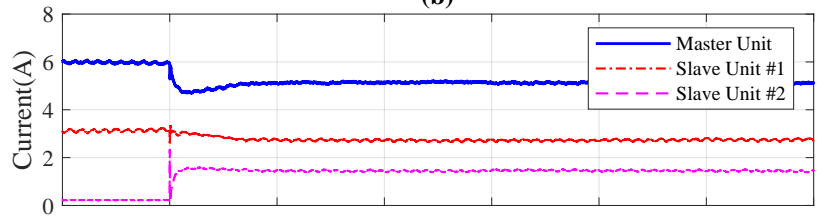

(c)

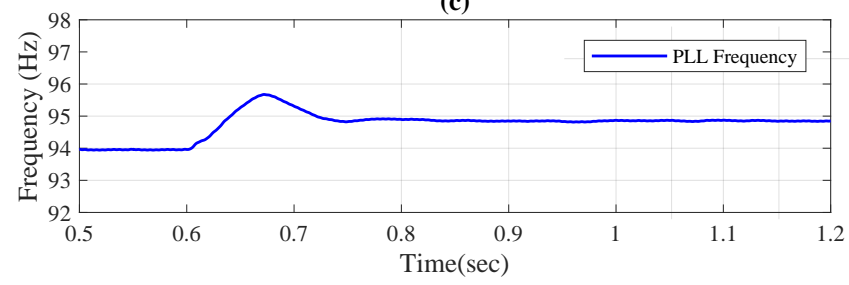

Fig. 12. Experimental results of the proposed control system while connecting the slave units: (a) DC bus voltage, (b) output current of the DG units, and (c) captured frequency by the first slave DG unit.

their ratings, and there is no voltage drop while sharing load. As it is illustrated, the master DG unit increases its output current from $2.97 A$ to $5.13 A$, the first slave DG unit experiences a rise in its output current from $1.46 A$ to $2.74 A$, and DG unit \#3 changes its output current from $0.81 A$ to $1.40 A$. This test confirms the load sharing accuracy. In addition, Fig. 13 (c) shows the captured superimposed frequency by the first slave DG unit, which confirms the correct performance of the proposed control system.

3) Master DG Unit Failure: The master DG unit outage is tested, while the DG units just supply their local loads. The centralized load is not connected to the DC MG to avoid overloading the DG units after the outage. Similar to the simulation results, the second DG unit is expected to change its role to the new master one. Figs. 14(a) and (b) depict the network DC bus voltage and the DG units output current, respectively. At $t=0.6 \mathrm{~s}$, the master unit is disconnected. According to the shown results, the network voltage is regulated, and the load sharing among the remaining DG units is still accurate. This test proves that the PnP feature is applicable for the master DG unit as well. In Fig. 14(c), the superimposed frequency of the network is shown. The maximum frequency drop is set to be $6 \mathrm{~Hz}$ for the network. According to the shown results, this superimposed frequency is $94 \mathrm{~Hz}$ for the network when the master DG unit is out, and all the slave DG units are synchronized to each other. (a)

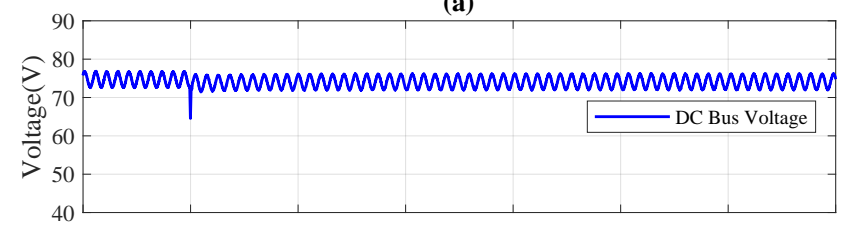

(b)

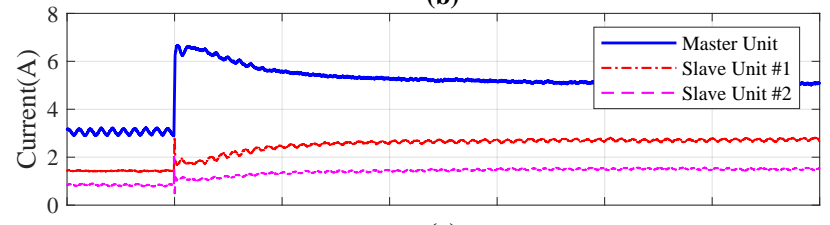

(c)

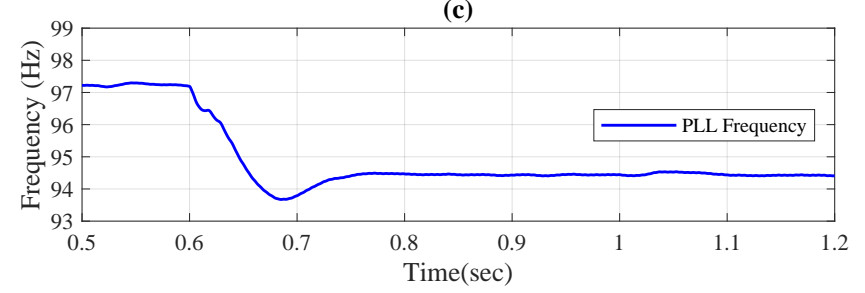

Fig. 13. Experimental results of the proposed control system in the presence of the centralized load connection: (a) DC bus voltage, (b) output current of the DG units, and (c) captured frequency by the first slave DG unit.
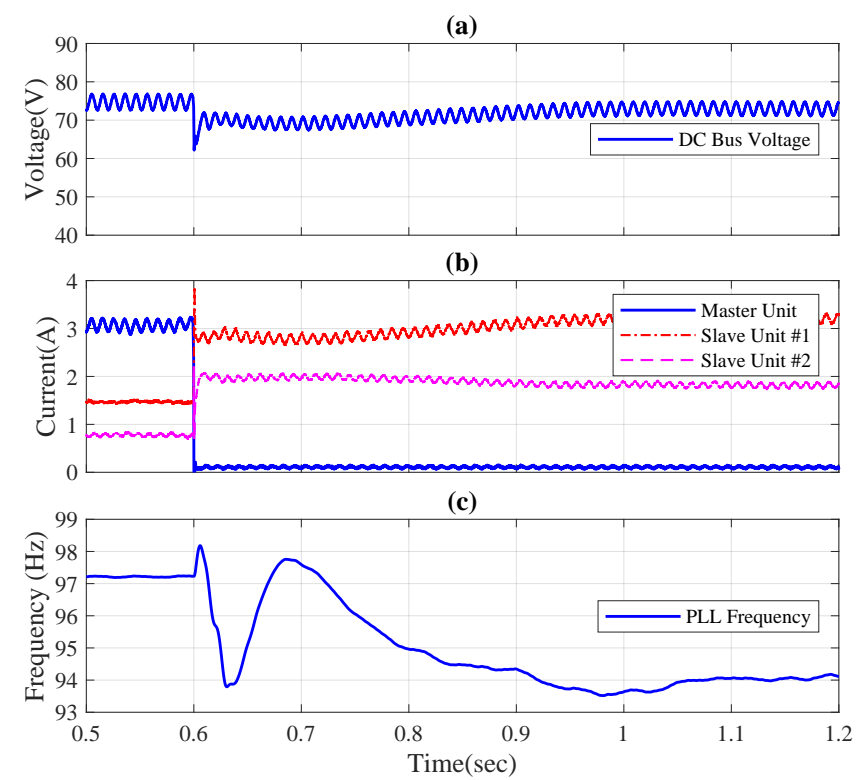

Fig. 14. Experimental results of the proposed control system facing the master DG unit outage: (a) DC bus voltage, (b) output current of the DG units, and (c) captured frequency by the first slave DG unit.

\section{Conclusions}

This paper proposes a control system for islanded DC MGs to share loads among DC DG units with respect to their nominal ratings. In load sharing, the proposed control system eliminates the effect of distribution line parameters and network structure without any communication between DG units. As a result, a decentralized control system is employed. Not only the decentralized control system provides PnP feature for the DG units, but also lack of communication lines increases the reliability of the network operation. The proposed control system compensates the voltage changes in the conventional droop control by modifying this technique. The compensation term shifts the terminal voltage of all DG units simultaneously using an identified value to avoid any instability. The voltage compensation term is adjusted 
using a superimposed $\mathrm{AC}$ voltage on the nominal $\mathrm{DC}$ voltage. The frequency of the superimposed $\mathrm{AC}$ voltage is proportional to the master DG unit's loading, i.e. the output current. The performance of the proposed control system is not dependant on the nominal frequency of the superimposed $\mathrm{AC}$ voltage. In this study, a frequency range between $90 \mathrm{~Hz}$ to $100 \mathrm{~Hz}$ is selected. The proposed control system for islanded DC MGs is verified using simulation studies via PLECS and a set of experiments.

\section{REFERENCES}

[1] J. J. Justo, F. Mwasilu, J. Lee, and J.-W. Jung, "AC-microgrids versus DCmicrogrids with distributed energy resources: A review," Renewable and Sustainable Energy Reviews, vol. 24, pp. 387-405, 2013.

[2] A. T. Elsayed, A. A. Mohamed, and O. A. Mohammed, "DC microgrids and distribution systems: An overview," Electric Power Systems Research, vol. 119, pp. 407-417, 2015.

[3] D. Boroyevich, I. Cvetkovic, R. Burgos, and D. Dong, "Intergrid: A future electronic energy network?" IEEE Journal of Emerging and Selected Topics in Power Electronics, vol. 1, no. 3, pp. 127-138, 2013.

[4] M. D'Antonio, C. Shi, B. Wu, and A. Khaligh, "Design and optimization of a solar power conversion system for space applications," IEEE Transactions on Industry Applications, vol. 55, no. 3, pp. 2310-2319, 2019.

[5] A. Accetta and M. Pucci, "Energy Management System in DC Micro-Grids of Smart Ships: Main Gen-Set Fuel Consumption Minimization and Fault Compensation," IEEE Transactions on Industry Applications, vol. 55, no. 3 , pp. 3097-3113, 2019.

[6] M. S. Sadabadi, Q. Shafiee, and A. Karimi, "Plug-and-play robust voltage control of DC microgrids," IEEE Transactions on Smart Grid, vol. 9, no. 6, pp. 6886-6896, 2018.

[7] J. M. Guerrero, J. C. Vasquez, J. Matas, L. G. De Vicuña, and M. Castilla, "Hierarchical control of droop-controlled AC and DC microgrids-A general approach toward standardization," IEEE Transactions on industrial electronics, vol. 58, no. 1, pp. 158-172, 2010.

[8] D.-H. Dam and H.-H. Lee, "A power distributed control method for proportional load power sharing and bus voltage restoration in a dc microgrid," IEEE Transactions on Industry Applications, vol. 54, no. 4, pp. 3616-3625, 2018.

[9] V. Nasirian, A. Davoudi, F. L. Lewis, and J. M. Guerrero, "Distributed adaptive droop control for DC distribution systems," IEEE Transactions on Energy Conversion, vol. 29, no. 4, pp. 944-956, 2014.

[10] F. Guo, Q. Xu, C. Wen, L. Wang, and P. Wang, "Distributed secondary control for power allocation and voltage restoration in islanded DC microgrids," IEEE Transactions on Sustainable Energy, vol. 9, no. 4, pp. 1857-1869, 2018.

[11] J. P. Lopes, C. Moreira, and A. Madureira, "Defining control strategies for microgrids islanded operation," IEEE Transactions on power systems, vol. 21, no. 2, pp. 916-924, 2006.

[12] W. Jiang and B. Fahimi, "Active current sharing and source management in fuel cell-battery hybrid power system," IEEE Transactions on Industrial Electronics, vol. 57, no. 2, pp. 752-761, 2009.

[13] S. Anand, B. G. Fernandes, and J. Guerrero, "Distributed control to ensure proportional load sharing and improve voltage regulation in low-voltage DC microgrids," IEEE Transactions on power electronics, vol. 28, no. 4, pp. 1900-1913, 2012.

[14] A. Khorsandi, M. Ashourloo, and H. Mokhtari, "A decentralized control method for a low-voltage DC microgrid," IEEE Transactions on Energy Conversion, vol. 29, no. 4, pp. 793-801, 2014.

[15] A. P. N. Tahim, D. J. Pagano, E. Lenz, and V. Stramosk, "Modeling and stability analysis of islanded DC microgrids under droop control," IEEE Transactions on power electronics, vol. 30, no. 8, pp. 4597-4607, 2014.

[16] S. Augustine, M. K. Mishra, and N. Lakshminarasamma, "Adaptive droop control strategy for load sharing and circulating current minimization in low-voltage standalone DC microgrid," IEEE Transactions on Sustainable Energy, vol. 6, no. 1, pp. 132-141, 2014

[17] J. Hu, J. Duan, H. Ma, and M.-Y. Chow, "Distributed adaptive droop control for optimal power dispatch in DC microgrid," IEEE Transactions on Industrial Electronics, vol. 65, no. 1, pp. 778-789, 2017.

[18] A. Khorsandi, M. Ashourloo, H. Mokhtari, and R. Iravani, "Automatic droop control for a low voltage DC microgrid," IET Generation, Transmission \& Distribution, vol. 10, no. 1, pp. 41-47, 2016.

[19] P. Prabhakaran, Y. Goyal, and V. Agarwal, "Novel Nonlinear Droop Control Techniques to Overcome the Load Sharing and Voltage Regulation Issues in DC Microgrid," IEEE Transactions on Power Electronics, vol. 33, no. 5, pp. 4477-4487, 2018.
[20] M. Nabatirad, B. Bahrani, and R. Razzaghi, "Decentralized Secondary Controller in Islanded DC Microgrids to Enhance Voltage Regulation and Load Sharing Accuracy," in IEEE International Conference on Industrial Technology. IEEE, 2019, Conference Proceedings, pp. 1692-1697.

[21] S. Peyghami, H. Mokhtari, and F. Blaabjerg, "Decentralized Load Sharing in a Low-Voltage Direct Current Microgrid With an Adaptive Droop Approach Based on a Superimposed Frequency," IEEE Trans. Emerg. Sel. Topics Power Electron., vol. 5, no. 3, pp. 1205-1215, 2017.

[22] S. Peyghami, H. Mokhtari, P. C. Loh, P. Davari, and F. Blaabjerg, "Distributed primary and secondary power sharing in a droop-controlled LVDC microgrid with merged AC and DC characteristics," IEEE Transactions on Smart Grid, vol. 9, no. 3, pp. 2284-2294, 2018.

[23] S. Peyghami, P. Davari, H. Mokhtari, and F. Blaabjerg, "Decentralized Droop Control in DC Microgrids Based on a Frequency Injection Approach," IEEE Transactions on Smart Grid, vol. 10, no. 6, pp. 6782-6791, 2019.

[24] K. A. Alobeidli, M. H. Syed, M. S. El Moursi, and H. H. Zeineldin, "Novel coordinated voltage control for hybrid micro-grid with islanding capability," IEEE Transactions on Smart Grid, vol. 6, no. 3, pp. 1116-1127, 2014.

[25] M. A. Setiawan, A. Abu-Siada, and F. Shahnia, "A new technique for simultaneous load current sharing and voltage regulation in DC microgrids," IEEE Transactions on Industrial Informatics, vol. 14, no. 4, pp. 1403-1414, 2018.

[26] M. Nabatirad, R. Razzaghi, and B. Bahrani, "Decentralized Voltage Regulation in Islanded DC Microgrids in the Presence of Dispatchable and Non-Dispatchable DC Sources," in 2020 22nd European Conference on Power Electronics and Applications (EPE'20 ECCE Europe). IEEE, 2020, Conference Proceedings, pp. 1-10.

[27] — - "Decentralized Voltage Regulation and Energy Management of Integrated DC Microgrids Into AC Power Systems," IEEE Journal of Emerging and Selected Topics in Power Electronics, vol. 9, no. 2, pp. 1269-1279, 2021.

[28] M. Ciobotaru, R. Teodorescu, and V. G. Agelidis, "Offset rejection for PLL based synchronization in grid-connected converters," in Twenty-Third Annual IEEE Applied Power Electronics Conference and Exposition. IEEE, 2008, pp. 1611-1617.

[29] M. Tucci, S. Riverso, J. C. Vasquez, J. M. Guerrero, and G. Ferrari-Trecate, "A decentralized scalable approach to voltage control of DC islanded microgrids," IEEE Transactions on Control Systems Technology, vol. 24, no. 6, pp. 1965-1979, 2016.

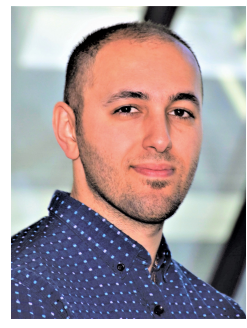

Mohammadreza Nabatirad (Graduate Student Member, IEEE) received the B.Sc. degree from Isfahan University of Technology, Isfahan, Iran, and the M.Sc. degree from Shahid Beheshti University, Tehran, Iran, both in Electrical Engineering - Power, in 2010 and 2012, respectively. Currently he is pursuing the Ph.D. degree with Monash University, Melbourne, Australia. His research interests are Power System Operation, Distributed Energy Resources and Storage Systems, Renewable Energy Systems, and Microgrids.

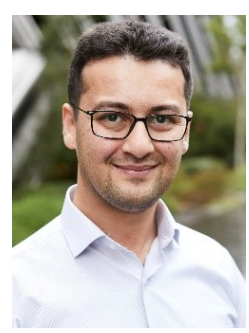

Reza Razzaghi (Member, IEEE) received the Ph.D degree in electrical engineering from the Swiss Federal Institute of Technology of Lausanne (EPFL), Lausanne, Switzerland in 2016. In 2017, he joined Monash University, Melbourne, Australia, where he is currently a Lecturer (Assistant Professor) with the Department of Electrical and Computer Systems Engineering. His research interests include power system protection and control, microgrids, and electromagnetic transients in power systems. He has been the recipient of the 2019 Best Paper Award of the IEEE Transactions on EMC and the 2013 Basil Papadias Best Paper Award from the IEEE PowerTech Conference. 


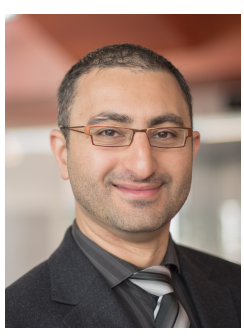

Behrooz Bahrani (Senior Member, IEEE) received the B.Sc. degree from Sharif University of Technology, Tehran, Iran, the M.Sc. degree from the University of Toronto, Toronto, ON, Canada, and the PhD degree from the Ecole Polytechnique Federale de Lausanne (EPFL), Lausanne, Switzerland, all in electrical engineering, in 2006, 2008, and 2012, respectively. From September 2012 to September 2015, he was a Postdoctoral Fellow at several institutions including EPFL, Purdue University,

West Lafayette, IN, USA, Georgia Institute of Technology, Atlanta, GA, USA, and the Technical University of Munich, Munich, Germany. Currently, he is a Senior Lecturer at Monash University, where he is also serving as the Director of the Grid Innovation Hub. His research interests include control of power electronics systems, applications of power electronics in power and traction systems, and grid integration of renewable energy resources. 